\title{
鳑鲏与河蚌交互作用对浮游生物和底栖生物的影响分析”
}

\author{
于谨磊 ${ }^{* *}$, 夏曼莉 ${ }^{2}$, 关保华 ${ }^{1,3}$,何 虎 $^{1}$, 陈非洲 ${ }^{1,3}$, 刘正文 ${ }^{1,2,3}$ \\ (1: 中国科学院南京地理与湖泊研究所湖泊与环境国家重点实验室，南京 210008) \\ (2: 暨南大学生态学系水生生物研究所, 广州 510632$)$ \\ (3: 中国科学院大学中丹科研教育中心, 北京 100049)
}

\begin{abstract}
摘 要: 放养河蛙, 提高水体透明度以促进沉水植物生长, 是湖泊生态修复中的常用手段之一. 而小型杂食性鱼类鳑鲏 依赖河蚌繁殖, 河蚌放养可能会促进鳑鲏种群的发展; 而鳑鲏与河蚌交互作用对水生态系统的影响仍研究较少. 于 2018 年 11-12 月通过原位受控实验, 设置对照组、河蚌组、鳑鲏组和河蚌+鳑鲏组, 研究了鳑鲏(大鯺鱊Acheilognathus macropterus) 与河蚌 (背角无齿蚌 Sinanodonta woodiana) 对水质、浮游生物和底栖生物的影响. 结果表明: 鳑鲏显著增加了上覆水 总氮、总磷、悬浮质和叶绿素 $a(\mathrm{Chl} . a)$ 浓度; 但并未显著影响无机悬浮质的浓度, 说明鳑鲏对沉积物的扰动作用较弱. 此 外, 鳑鲏显著增加了浮游植物生物量和蓝藻的比例. 浮游动物总生物量并未受鳑鲏的影响, 但显著增加了浮游动物的丰 度, 其中以轮虫为主. 鳑鲏组的底栖动物总丰度 (主要为水丝蚂) 显著高于对照组, 说明鳑鲏对水丝蚂的摄食压力较低. 鳑鲏与河蚌交互作用对总磷、Chl. $a$ 、浮游植物和浮游动物丰度具有显著影响: 具体表现为河蚌虽然抵消了部分鳑鲏对水 质 (如氮、磷和悬浮质浓度, 以及 Chl. $a$ 浓度和浮游植物生物量等) 的负面影响, 但其对浮游动物和水丝蝲生物量的影响 不显著. 在湖泊生态修复和管理中, 需要关注和重视鳑鲏等此类小型杂食性鱼类对水生态系统可能产生的负面影响, 通 过物理 (网箱等) 或生物 (放养肉食性鱼类) 等方式将其控制在较低密度水平, 从而降低这些鱼类对水质、浮游生物和底 栖生物群落可能产生的负面影响, 维持生态修复效果的长效与稳定运行.
\end{abstract}

关键词: 杂食性鱼类;营养盐; 浮游植物; 浮游动物; 水丝蚂; 大鯺鱊; 背角无齿蚌

\section{Interactive effects of bitterling fish and mussel on the community structures of plankton and benthic macroinvertebrates *}

Yu Jinlei ${ }^{1 * *}$, Xia Manli ${ }^{2}$, Guan Baohua ${ }^{1,3}, \mathrm{He} \mathrm{Hu}^{1}$, Chen Feizhou ${ }^{1,3}$ \& Liu Zhengwen ${ }^{1,2,3}$

(1: State Key Laboratory of Lake Science and Environment, Nanjing Institute of Geography and Limnology, Chinese Academy of Sciences, Nanjing 210008, P.R.China)

(2: Department of Ecology and Institute of Hydrobiology, Jinan University, Guangzhou 510632, P.R. China)

(3: Sino-Danish Center for Education and Research, University of Chinese Academy of Sciences, Beijing 100049, P.R.China)

\begin{abstract}
In restoration of shallow eutrophic lakes based on recovery of submerged macrophytes, stocking of the filter-feeding mussels has been commonly used for improving water clarity, thereby enhancing the growth of submerged macrophytes. However, the small omnivorous bitterling fish has to rely on mussels for breeding. Thus, the increased abundance of mussels may enhance the population development of bitterling. Nevertheless, the effects of bitterling (Acheilognathus macropterus) and their host mussels (Sinanodonta woodiana) on the aquatic ecosystems are, so far, not well-studied. We conducted a mesocosm experiment to study the effects of bitterling, mussels, and their interactions on water quality and the community structures of phytoplankton, zooplankton and benthic macroinvertebrates, respectively. Our results showed that bitterling did not substantially affect the concentration of inorganic suspended solids (ISS) indicating weak effects on sediment resuspension. However, the concentrations of total nitrogen and total phosphorus were significantly enhanced by bitterling. Consequently, both the abundance and biomass of phytoplankton were enhanced in the bitterling-only mesocosms, and cyanobacteria dominated the phytoplankton community at the end of the ex-
\end{abstract}

* 2020-09-02 收稿;2020-11-25 收修改稿.

国家自然科学基金项目 (41877415) 和国家水体污染控制与治理科技重大专项 (2017ZX07203-004)联合资助.

** 通信作者; E-mail: jlyu@ niglas.ac.cn. 
periment. However, rotifers dominated the zooplankton community leading to a higher abundance of zooplankton in the bitterlingonly treatment, while the total biomass of zooplankton did not differ significantly between the control and bitterling-only treatments. Moreover, bitterling significantly increased the total abundance of benthic macroinvertebrates compared with the controls, while the biomass of macroinvertebrates was not pronouncedly affected. The presence of mussels significantly eliminated the negative effects induced by bitterling, such as reducing the concentrations of nutrients, suspended solids and chlorophyll- $a$ and phytoplankton biomass. However, the biomass of zooplankton and benthic macroinvertebrates were not substantially affected by the presence of mussels. Our results suggest that bitterling alone can induce significantly negative effects on water quality though mussels can eliminate part of these impacts under our experimental bitterling-to-mussel biomass ratio. Therefore, in the restoration and management of shallow lakes, more attention should be paid on the effects of small omnivorous fish, like bitterling, on aquatic ecosystems. The biomass of these small omnivores should be controlled at a relatively low level by physical (e.g., trap net) or biological (e.g., stocking of effective piscivorous fish) methods to minimize the negative effects of the fish on lake ecosystems.

Keywords: Omnivorous fish; nutrient concentrations; phytoplankton; zooplankton; Limnodrilus ; Acheilognathus macropterus ; Sinanodonta woodiana

杂食性鱼类是指可同时摄食植物性饵料 (如沉水植物等) 和动物性饵料 (如底栖动物等) 的鱼类. 因此, 杂食性鱼类可从至少 2 个营养级中获取食物, 通过多种途径影响水生态系统. 底栖杂食性鱼类 (例如鲤和 鲫) 在取食底栖动物的过程中, 可显著引起水体营养盐浓度和浊度升高 ${ }^{[1-4]}$, 从而促进浮游植物生长和生物 量升高 ${ }^{[1,3]}$. 此外, 杂食性鱼类还可导致浮游动物和底栖动物生物量下降 ${ }^{[3,5]}$. 杂食性鱼类引起的浮游动物 生物量下降, 将削弱浮游动物对浮游植物生物量的下行控制力, 间接地促进了浮游植物生物量增长. 目前 有关杂食性鱼类的研究主要集中在个体较大的种类 (鲤、鲫等 $)^{[4,6-7]}$, 而对小型杂食性鱼类对水生态系统的 影响研究较少.

在五里湖生态修复区, 鳑鲏等小型杂食性鱼类是鱼类群落的优势鱼类 ${ }^{[8]}$. 鳑鲏是鲤科鱊亚科鱼类的统 称, 在我国分布广泛 ${ }^{[9]}$, 属于小型杂食性鱼类, 可摄食浮游动物、底栖动物、附着藻类、碎屑和沉水植物 等 ${ }^{[8,10]}$. 鳑鲏可能与其他杂食性鱼类 (例如鲤和鲫) 类似, 主要通过扰动沉积物 ${ }^{[3-4]}$ 、排泄 ${ }^{[11]}$ 、捕食浮游动 物 ${ }^{[12]}$ 、底栖动物 ${ }^{[3]}$ 和沉水植物 ${ }^{[8]}$ 等途径, 影响水质和生物群落结构. 但鳑鲏对水生态系统影响的研究还相 对较少 ${ }^{[13-14]}$.

鳑鲏繁殖时必需依赖河蚌 (例如背角无齿蚌 (Sinanodonta woodiana) 和三角帆蚌 (Hyriopsis cumingii) 等 ${ }^{[15]}$ ), 其受精卵在河蚌体内孵化, 幼鱼在河蚌外套膜生活一段时间后才独自生活 ${ }^{[16-17]}$. 河蚌通过滤食可 有效提高水体透明度 ${ }^{[18-20]}$, 促进沉水植物生长 ${ }^{[5]}$. 因此, 在以构建沉水植物群落为主的富营养化湖泊生态 修复中, 常将放养河蚌作为沉水植物群落恢复的辅助手段 ${ }^{[21-23]}$. 而放养的河蚌可能通过排泄营养盐 ${ }^{[24]}$ 和促 进鳑鲏种群的发展 (携带鳑鲏的卵或为已有鳑鲏种群提供产卵基质) 等途径影响水生态系统.

鳑鲏可引起水体营养盐浓度、浮游植物生物量和悬浮质浓度升高 ${ }^{[14]}$, 而河蚌通过滤食可能会缓解 (抵 消) 鳑鲏对水质造成的这些负面影响. 现有研究主要从鳑鲏一河蚌协同进化的角度研究两者间的相互作 用 ${ }^{[25-26]}$, 而它们之间的交互作用对水生态系统的影响还尚不清楚. 本研究以大䲠䁤 (Acheilognathus macropterus) (鳑鲔) 和背角无齿蚌 (河蚌) 为研究对象, 通过受控实验探讨鳑鲏和河蛙及其交互作用对水质以及浮 游植物、浮游动物和底栖动物群落结构的影响, 为我国浅水湖泊管理与生态修复提供参考.

\section{1 材料与方法}

\section{1 实验设置}

本实验于 2018 年 11- 12 月, 在中国科学院太湖湖泊生态系统研究站中设置 12 个高密度聚乙烯桶实 验系统 (底部直径: $43 \mathrm{~cm}$; 开口直径 $51 \mathrm{~cm}$; 桶高: $70 \mathrm{~cm}$; 容积: $130 \mathrm{~L}$ ). 实验开始前, 向每个实验系统中加 人 $10 \mathrm{~cm}$ 混匀的沉积物 (经孔径为 $0.5 \mathrm{~cm}$ 的篎网过滤) 以及 $100 \mathrm{~L}$ 经 $64 \mu \mathrm{m}$ 浮游生物网过滤的湖水. 沉积物 和水均采自实验站的池塘.

本实验共设置 4 个处理组, 分别为对照组、河蚌组、鳑鲏组和河蚌+鳑鲏组, 每个处理组设置 3 个重复.

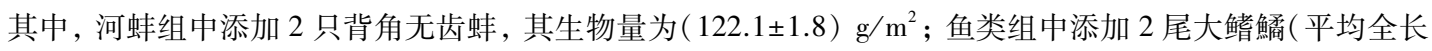


$(4.6 \pm 0.1) \mathrm{cm}$; 平均生物量 $\left.(12.8 \pm 0.2) \mathrm{g} / \mathrm{m}^{2}\right)$; 河蚌+鳑鲏组中添加 2 只河蚌 $\left(\right.$ 生物量 $\left.(120.6 \pm 0.7) \mathrm{g} / \mathrm{m}^{2}\right)$ 和 2 尾大鯺鱊 (平均全长 $(4.4 \pm 0.02) \mathrm{cm}$; 平均生物量 $(12.1 \pm 0.1) \mathrm{g} / \mathrm{m}^{2}$ ) ; 对照组中不添加河蚌和大鯺鱊. 本实 验采用的鱼类密度 $\left(3.8 \mathrm{ind} . / \mathrm{m}^{2}\right)$ 小于自然湖泊中的小型鱼类密度 $\left(8.7 \mathrm{ind} . / \mathrm{m}^{2}\right)^{[27]}$, 河蚌的生物量低于 $2006-2007$ 年太湖蚌类的总平均生物量 $\left(647 \mathrm{~g} / \mathrm{m}^{2}\right)^{[28]}$. 鳑鲏和河蚌均采自太湖, 并在实验开始时直接投放 到相应的系统.

\section{2 样品采集与分析}

实验开始后, 每 7 天测定一次水体的营养盐、悬浮质和叶绿素 $a(\mathrm{Chl} . a)$ 浓度. 实验结束时, 分析浮游植 物、浮游动物和底栖动物的丰度和生物量. 每次水样采集时, 用 $2.5 \mathrm{~L}$ 的有机玻璃采水器, 收集 $5 \mathrm{~L}$ 不同水 层的上覆水, 然后带 $2 \mathrm{~L}$ 混合均匀的水样回实验室分析水体不同形态的氮、磷营养盐浓度、Chl. $a$ 浓度和不 同形态的悬浮物浓度; 然后将剩余水样倒回实验系统. 浮游植物和浮游动物样品采集时, 用采水器收集 7.5 $\mathrm{L}$ 水样, 然后用 $8 \%$ 的鲁哥试剂固定 $500 \mathrm{~mL}$ 水样, 用于分析浮游植物的群落结构组成; 随后, 通过 $64 \mu \mathrm{m}$ 的浮游生物网过滤 $5 \mathrm{~L}$ 混合水样获取浮游动物群落样品, 并保存于 $4 \%$ 的甲醛溶液; 最后, 将剩余的水样和 经浮游动物网过滤的水倒人实验系统. 每次采样结束或当系统水位变化明显时, 用提前准备好的湖水将水 位恢复至实验初始水平. 在实验结束时, 用彼得森采泥器采集沉积物中的底栖动物. 浮游植物在 $100 \sim 400$ 倍显微镜下, 鉴定到属 ${ }^{[29-30]}$; 不同浮游植物体积的 0.29 倍作为各类植物的干重生物量 ${ }^{[31]}$. 浮游动物样品分 析时, 轮虫在 100 倍视野中鉴定, 枝角类和桡足类则在 $10 \sim 40$ 倍视野中进行鉴定 ${ }^{[32-33]}$, 然后根据浮游动物 体长与干重的经验公式计算浮游动物的干重生物量 ${ }^{[34-35]}$.

总氮 (TN)、溶解性总氮 (TDN)、总磷 (TP)、溶解性总磷 (TDP) 浓度的分析方法参照《湖泊富营养化调查 规范》 ${ }^{[36]}$. 测定水体 Chl. $a$ 浓度时, 用醋酸纤维膜过滤 $0.5 \sim 1 \mathrm{~L}$ 水样, 将滤膜转人离心管, 加人 $10 \mathrm{~mL} 90 \%$ 的丙酮溶液, 充分震荡后置于 $4^{\circ} \mathrm{C}$ 暗处反应 $18 \sim 24 \mathrm{~h}$; 随后, 经 $3500 \mathrm{r} / \mathrm{min}$ 离心后, 取上清液, 用紫外分光 光度计测定其在 750、663、645 和 $630 \mathrm{~nm}$ 波长下的吸光值, 计算水样中的 Chl. $a$ 浓度.

用已知重量的 Whatman $\mathrm{GF} / \mathrm{C}$ 玻璃纤维摸抽滤 $0.5 \sim 1 \mathrm{~L}$ 水样, 随后将膜置于 $60^{\circ} \mathrm{C}$ 烘箱内烘至恒重(约 $48 \mathrm{~h}$ ), 称量后获得总悬浮质 ( TSS) 浓度. 随后, 将膜置于 $450^{\circ} \mathrm{C}$ 的马弗炉中灼烧 $4 \mathrm{~h}$, 取出称重, 获得有机悬 浮质 (OSS) 浓度; TSS 与 OSS 的差值即为无机悬浮质 (ISS) 浓度.

\section{3 数据分析}

本研究的所有数据统计分析均在 SPSS 22.0 软件中进行. 通过双因素 (鳑鲏和河蚌) 重复测量方差分析 方法 (rmANOVA)，分析鳑鲏、河蚌以及鳑鲏与河蚌交互作用对 TN、TDN、TP、TDP、Chl. $a$ 、TSS、OSS、ISS 浓度 的影响; 通过双因素方差分析, 比较了实验结束时不同处理组间浮游植物、浮游动物和底栖动物的影响. 所 有实验数据经 Kolmogorov-Smirnov 正态性检验和 Levene's 方差齐性检验, 必要时对数据进行 $\lg x$ 转换. 其中 组内比较参照一元方差分析中 Greenhouse-Giesser 校正后的结果; 组间比较参照 Tukey post-hoc 方法的分析 结果.

\section{2 结果}

\section{1 上覆水营养盐}

实验期间，鳑鲏与河蚌对水体 $\mathrm{TN}$ 浓度的影响显著，而两者交互作用对 $\mathrm{TN}$ 浓度的影响不显著 (表 1$)$. 与对照组相比, 鳑鲏显著增加了水体 $\mathrm{TN}$ 浓度 $(F=8.7, P=0.019)$; 而河蚌组的 $\mathrm{TN}$ 浓度与对照组相近 $(F=$ $0.1, P=0.7$; 图 $1 \mathrm{~A}$ ). 当河蚌与鳑鲏同时存在时, 河蚌的出现显著降低了鳑鲏对水体 $\mathrm{TN}$ 浓度的影响 (鳑鲏+ 河蚌组 vs 鳑鲏组, $F=8.6, P=0.019$; 图 1A). 此外, 鳑鲏+河蚌组的水体 $\mathrm{TN}$ 浓度与河蚌组间无显著性差异 $(F=0.1, P=0.7)$. 水体 TDN 浓度并未受鳑鲏、河蚌以及两者交互作用的影响 (表 1). 同时, 各实验处理组 间的 TDN 浓度也均无显著差异(图 1B).

河蛙、鳑鲏以及两者的交互作用对水体 TP 浓度具有显著的影响 (表 1). 鳑鲏组的 TP 浓度显著高于对 照组 ( $F=22.2, P=0.002)$, 低于鳑鲏+河蚌组 ( $F=18.8, P=0.002$; 图 2). 河蚌组的 TP 浓度与对照组和河 蚌+鳑鲏组间均无显著差异 (图 1C). 此外, 仅鳑鲏对水体 TDP 浓度的影响显著, 河蚌以及鳑鲏与河蚌的交 互作用对 TDP 浓度的影响均不显著 (表 1). 鳑鲏组的 TDP 浓度显著高于对照组 $(F=11.9, P=0.009$; 图 
1D). 河蚌组的 TDP 与对照组和鳑鲏+河蚌组间均无显著差异 ( 图 1D).

\section{2 悬浮质}

实验期间, 河蚌和鳑鲏对 TSS 浓度的影响显著, 而两者的交互作用对 TSS 浓度的影响不显著 (表 1). 鳑鲏组的 TSS 浓度显著高于对照组 $(F=20.9, P=0.002)$ 和鳑鲏+河蚌组 $(F=23.2, P=0.001)$; 而河蚌组与 对照组以及鳑鲏+河蚌组间的 TSS 浓度均无显著差异 (图 2A). 鳑鲏显著引起 OSS 浓度升高, 其中鳑鲏组的 OSS 浓度显著高于对照组 $(F=17.95, P=0.003)$; 而河蚌的出现可显著降低鳑鲏引起的 OSS 浓度现象, 鳑 鲏+河蚌组的 OSS 浓度显著低于鳑鲏组 $(F=25.03, P=0.001)$. 河蚌出现的处理组中, 无论是否有鳑鲏出 现, 其各处理组间的 OSS 浓度均无显著差异 (图 2B). 此外, 鳑鲏与河蛙的交互作用也并未显著影响 OSS 浓 度 (表 1). 鳑鲏、河蛙、鳑鲏与河蚌的交互作用对 ISS 浓度均无显著影响 (表 1), 其中各实验处理组间的 ISS 浓度均无显著差异 $(P>0.05$; 图 2C).

\section{表 1 鳑鲏与河蚌对水体 TN、TDN、TP、TDP、TSS、OSS、ISS 和 Chl. $a$ 浓度影响的 双因子重复测量方差分析结果 (rmANOVA)}

Tab.1 Results of rmANOVA on the effects of mussel and bitterling treatments on total nitrogen ( TN), total dissolved nitrogen ( TDN), total phosphorus ( TP), total dissolved phosphorus ( TDP), total suspended solids (TSS), total organic suspended solids ( OSS), total inorganic suspended solids ( ISS), and chlorophyll- $a$ (Chl. $a$, phytoplankton biomass) concentrations during the mesocosm experiment

\begin{tabular}{|c|c|c|c|c|c|c|c|c|}
\hline 指标 & 统计参数 & 鳑鲏 & 河蚌 & 鳑鲏×河蚌 & 时间 & 时间 $\times$ 鳑鲏 & 时间 X河蚌 & 时间 $\times$ 鳑鲏 $\times$ 河蚌 \\
\hline \multirow[t]{2}{*}{$\mathrm{TN}$} & $F$ & 5.5 & 5.5 & 3.3 & 10.9 & 2.7 & 2.8 & 1.6 \\
\hline & $P$ & 0.047 & 0.048 & 0.11 & $<0.0001$ & 0.014 & 0.009 & 0.14 \\
\hline \multirow[t]{2}{*}{ TDN } & $F$ & 3.0 & 0.5 & 2.7 & 15.1 & 3.4 & 2.2 & 3.3 \\
\hline & $P$ & 0.12 & 0.49 & 0.14 & $<0.0001$ & 0.02 & 0.086 & 0.021 \\
\hline \multirow[t]{2}{*}{ TP } & $F$ & 16.7 & 12.7 & 6.6 & 14.5 & 5.2 & 3.3 & 2.1 \\
\hline & $P$ & 0.004 & 0.007 & 0.033 & $<0.0001$ & 0.014 & 0.053 & 0.15 \\
\hline \multirow[t]{2}{*}{ TDP } & $F$ & 9.4 & 2.5 & 3.3 & 11.4 & 4.2 & 1.6 & 3.1 \\
\hline & $P$ & 0.015 & 0.15 & 0.11 & $<0.0001$ & 0.02 & 0.23 & 0.052 \\
\hline \multirow[t]{2}{*}{ TSS } & $F$ & 18.8 & 22 & 4.5 & 96.9 & 3.1 & 6.7 & 2.4 \\
\hline & $P$ & 18.8 & 0.002 & 0.066 & $<0.0001$ & 0.005 & $<0.0001$ & 0.025 \\
\hline \multirow[t]{2}{*}{ OSS } & $F$ & 15.3 & 23.7 & 4.9 & 27.9 & 2.9 & 3.7 & 2.5 \\
\hline & $P$ & 0.005 & 0.001 & 0.058 & $<0.0001$ & 0.009 & 0.001 & 0.019 \\
\hline \multirow[t]{2}{*}{ ISS } & $F$ & 5.7 & 1.4 & 0.02 & 75.3 & 1.2 & 2.1 & 0.8 \\
\hline & $P$ & 0.054 & 0.28 & 0.90 & $<0.0001$ & 0.32 & 0.12 & 0.54 \\
\hline \multirow[t]{2}{*}{ Chl. $a$} & $F$ & 20.4 & 16.4 & 11.9 & 4.6 & 6.1 & 5 & 4.5 \\
\hline & $P$ & 0.002 & 0.004 & 0.009 & 0.037 & 0.018 & 0.030 & 0.040 \\
\hline
\end{tabular}

\section{3 浮游植物}

鳑鲏、河蚌以及两者的交互作用均可显著影响水体 Chl. $a$ 浓度 (表 1). 实验期间, 鳑鲏组的 Chl. $a$ 浓度 显著高于对照组 $(F=31.8, P<0.0001)$. 而当河蚌出现时, 鳑鲏+河蚌组的 Chl. $a$ 浓度显著低于鳑鲏组 ( $F=$ 28.1, $P=0.001)$. 河蚌组与对照组 $(F=0.2, P=0.7)$ 和鳑鲏+河蚌组 $(F=0.6, P=0.5)$ 的 Chl. $a$ 浓度均无显 著差异 (图 3).

实验结束时, 与对照组相比, 鳑鲏显著增加了浮游植物的丰度 $(F=7.0, P=0.029)$ 和生物量 $(F=7.0$, $P=0.03)$. 河蛙显著抑制了鳑鲏导致的浮游植物丰度和生物量增长, 表现为鳑鲏+河蚌组的浮游植物丰度 $(F=13.7, P=0.006)$ 和生物量 $(F=24.7, P=0.001)$ 均显著低于鳑鲏组 (图 4). 此外, 河蚌组与对照组间的 浮游植物也无显著差异 ( 丰度 $F=2.6, P=0.14$; 生物量 $F=3.5, P=0.1$ ).

鳑鲏组的浮游植物以蓝藻门为主 (优势种为束丝藻 Aphanizomenon), 占总生物量的 $63 \% \pm 28 \%$. 而河蚌 的出现 (鳑鲏+河蚌组)，降低了蓝藻门的比例 ( $24 \% \pm 41 \%)$; 硅藻门 ( $37 \% \pm 21 \%$ ) 和隐藻门 ( $34 \% \pm 31 \%$ ) 的 种类为该处理组的优势种, 其中硅藻门以针杆藻 Synedra 为主、隐藻门以蓝隐藻 Chroomonas 为主. 对照组和 

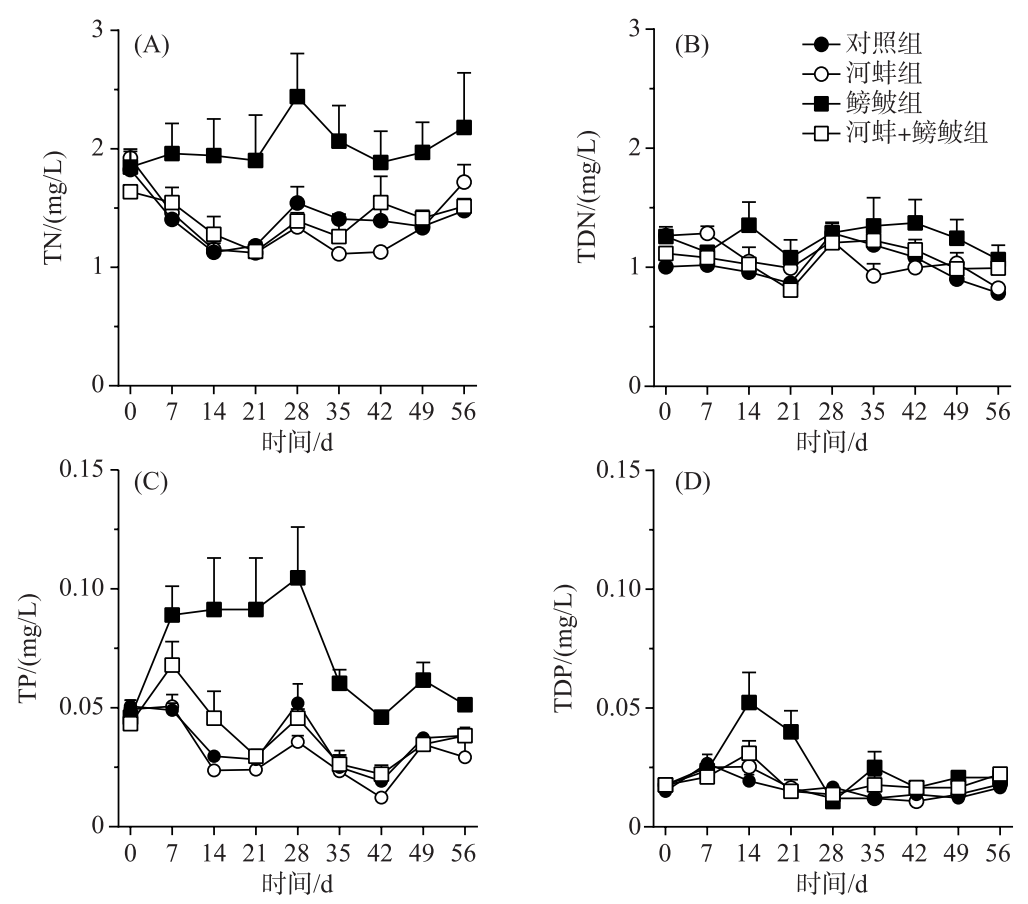

图 1 不同处理组的总氮、总溶解性氮以及总磷、总溶解性磷浓度随时间的变化趋势

Fig. 1 Concentrations of total nitrogen, total dissolved nitrogen, total phosphorus and total dissolved phosphorus in different treatments during the experiment


图 2 不同处理组的总悬浮质 (TSS)、有机悬浮质 ( OSS) 和无机悬浮质 (ISS) 浓度随时间的变化

Fig.2 Concentrations of total suspended solids (TSS), organic suspended solids ( OSS) and inorganic suspended solids (ISS) in different treatments during the experiment

河蚌组的优势种均为隐藻门的隐藻, 分别占各组生物量的比例为 $48 \% \pm 41 \%$ 和 $56 \% \pm 51 \%$.

\section{4 浮游动物}

鳑鲔显著增加了浮游动物的丰度 $(F=17.9, P=0.003)$, 而对浮游动物生物量无显著影响 $(F=0.06, P=$ $0.80)$. 河蚌对浮游动物丰度 $(F=5.03, P=0.06)$ 和生物量 $(F=1.2, P=0.31)$ 的影响均不显著. 鳑鲔与河蚌 的交互作用对浮游动物丰度的影响显著 $(F=6.7, P=0.032)$, 但对总生物量无显著影响 $(F=0.2, P=0.64)$.

实验结束时, 对照组中检出个体较大的枝角类 (如长肢秀体溞 Diaphanosoma leuchtenbergianum 和透明 薄皮渪 Leptodora kindti)，但在鳑鲏出现的实验组中未检出枝角类. 而鳑鲏组的浮游动物丰度显著高于对照 


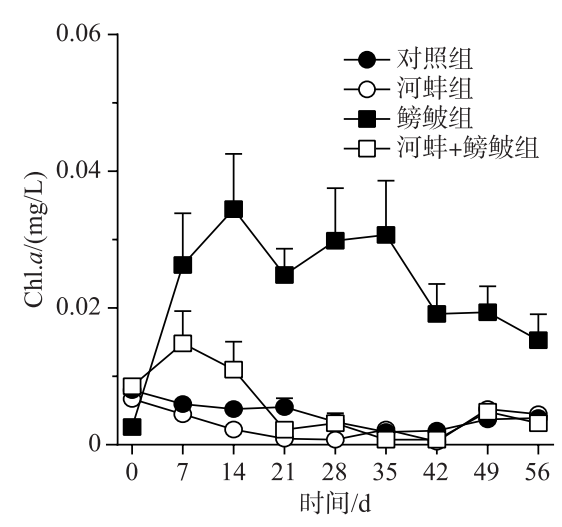

图 3 不同处理组的水体 Chl. $a$ 浓度

Fig. 3 Concentrations of chlorophyll- $a$ in different treatments during the experiment
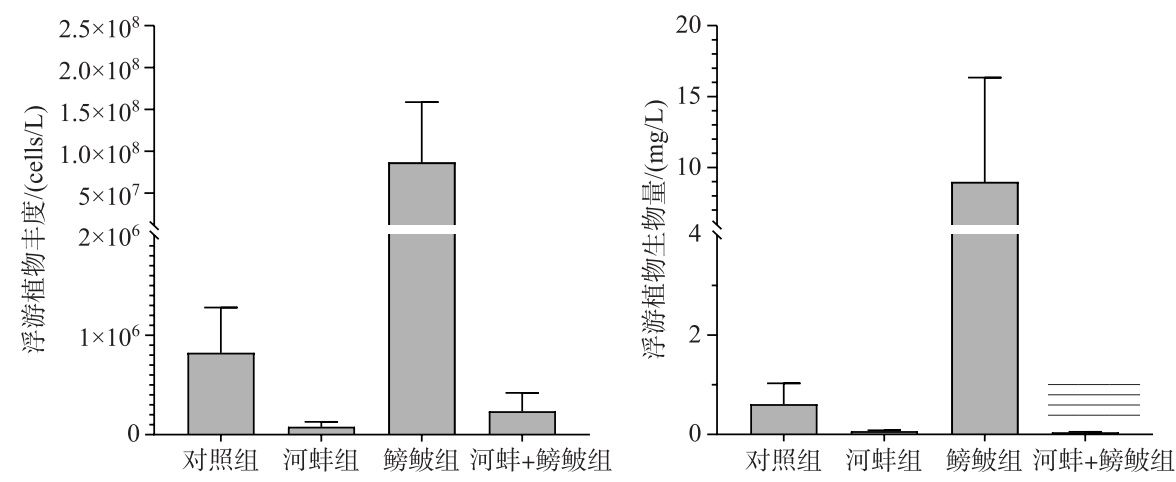

图 4 实验结束时不同处理组的浮游植物丰度和生物量

Fig.4 The abundance and biomass of phytoplankton in different treatments at the end of the experiment

组 $(F=23.4, P=0.001)$, 生物量与对照组间无显著差异 $(F=0.3, P=0.62)$. 鳑鲏组以轮虫为主 (优势种为螺 形龟甲轮虫 Keratella cochlearis 和月型腔轮虫 Lecane buna), 未检测到枝角类. 河蚌组与鳑鲏+河蚌组间的丰 度 $(F=1.4, P=0.28)$ 和生物量 $(F=0.03, P=0.87)$ 均无显著差异 (图 5). 河蚌组的浮游动物总丰度显著高于 对照组 ( $F=11.7, P=0.009)$, 而这 2 个处理组的生物量相近 $(F=1.2, P=0.3)$. 鳑鲏组与鳑鲏+河蚌组的浮 游动物丰度 $(F=0.06, P=0.8)$ 和生物量 $(F=0.2, P=0.7)$ 均无显著差异 ( 图 5$)$.

\section{5 底栖动物}

鳑鲏显著促进了底栖动物丰度上升 $(F=10.1, P=0.013)$, 而对生物量的影响不显著 (图 6$)$; 河蛙、鳑鲏 与河蚌的交互作用对底栖动物的丰度和生物量均无显著影响 $(P>0.05)$.

实验结束时, 鳑鲏组的底栖动物总丰度显著高于对照组 $(F=8.04, P=0.022)$, 而两组间的生物量却无 显著差异 $(F=4.9, P=0.058)$. 河蚌组与对照组 $(P>0.05)$ 、河蚌组与鳑鲏+河蚌组 $(P>0.05)$ 、鳑鲏组与鳑鲏+ 河蚌组 $(P>0.05)$ 间的底栖动物丰度和生物量均无显著差异 (图 6 ).

\section{3 讨论}

在本研究中, 大鯺鱊 (以下简称鳑鲏)引起水体营养盐浓度升高、浮游动物以小型种类轮虫为主、枝角 类生物量下降. 鳑鲏驱动的营养盐供给增加与捕食压力下降, 最终导致浮游植物生物量显著升高, 增加了 蓝藻的占比. 鳑鲏对 ISS 浓度无显著影响, 说明鳑鲏对沉积物的扰动作用较弱; 而鳑鲏可显著增加 OSS 和 TSS 浓度, 这说明鳑鲏主要通过影响 OSS 浓度的途径增加 TSS 浓度. 此外, 鳑鲏组的底栖动物总丰度显著 



图 5 实验结束时不同处理组的浮游动物丰度与生物量

Fig. 5 The abundance and biomass of zooplankton in different treatments at the end of the experiment
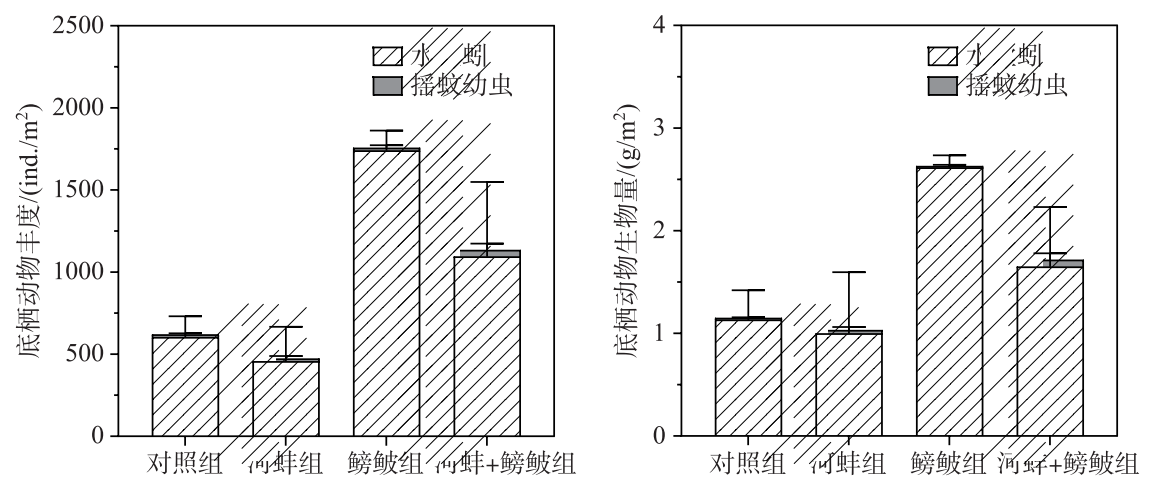

图 6 实验结束时不同处理组底栖动物丰度和生物量

Fig. 6 The abundance and biomass of benthic macroinvertebrates in different treatments at the end of the experiment

高于对照组, 主要以水丝蚓为主.

背角无齿蚌抵消了部分鳑鲏对水生态系统造成的这些负面作用 (如降低水体营养盐浓度、抑制浮游植 物生物量上升等). 鳑鲏与河蚌的交互作用对水体 TP、Chl. $a$ 、浮游植物生物量和浮游动物丰度的影响显著, 而对水体 TN、TSS、浮游动物生物量以及底栖动物丰度和生物量均无显著影响.

\section{1 大鯺鱊对水体理化因子的影响分析}

杂食性鱼类可通过排泄和扰动沉积物等方式影响水体营养盐浓度 ${ }^{[6,37-38]}$, 其主要影响途径与鱼类的体 型和摄食习性有关. 底栖杂食性鱼类体型较大, 主要以底栖动物为食 (如摇蚊幼虫和水丝蚓), 其摄食活动 可显著促进沉积物再悬浮, 增加水体无机悬浮质的浓度. 鱼类对沉积物的扰动越强, 其无机悬浮质的浓度 也就越高. 因此, 无机悬浮质浓度常被用于评价鱼类对沉积物再悬浮的影响程度. 本研究中, 鳑鲏组的营养 盐浓度显著高于对照组, 而无机悬浮质浓度却无显著差异, 这说明鳑鲏对沉积物的扰动作用较弱. 鳑鲏组 中较高的氮、磷浓度则可能主要由鱼类排泄所致. 这与已有的鳑鲔鱼类 (中华鳑鲏或大鯺鱊) 对水体营养盐 影响的结果一致 ${ }^{[13-14]}$. 在本研究中, 鳑鲏可能主要通过摄食浮游动物、摇蚁幼虫和附着藻等途径加速营养 盐循环. 因为, 相较于对照组, 鳑鲏组中未检出枝角类和摇蚊幼虫, 桡足类生物量低于对照组. 此外, 鳑鲏 可摄食附着藻类 ${ }^{[10]}$, 这不但会减少附着藻类对水体营养盐的吸收, 通过摄食附着藻还会增加排泄营养盐, 最终导致水体营养盐浓度升高.

\section{2 大鯺鱊对浮游生物的影响分析}

底栖杂食性鱼类 (如鲫) 可降低浮游动物生物量 ${ }^{[6,12]}$. 本研究中, 鳑鲏不但对浮游动物总生物量的影响 
不明显, 而且还显著增加了浮游动物的总丰度, 其中主要以小型浮游动物 (轮虫) 为主. 鱼类选择性摄食个 体较大的浮游动物是引起浮游动物群落小型化的主要原因 ${ }^{[39]}$. 在自然湖泊中, 鳑鲏可摄食大量的浮游动 物 ${ }^{[8]}$. 在本研究中, 鳑鲏组中未检出枝角类, 这与其他杂食性鱼类的研究结果一致 ${ }^{[3]}$.

鳑鲏引起的氮、磷浓度升高和捕食压力下降 (小型浮游动物为主), 促进了浮游植物丰度和生物量的升 高, 改变了浮游植物群落结构. 在其他杂食性鱼类研究中, 鱼类的出现也显著增加了浮游植物的丰度和生 物量 ${ }^{[6,40-41]}$. 本研究中, 蓝藻门的束丝藻是鳑鲏组的绝对优势种, 而 $\mathrm{He}$ 等 ${ }^{[6]}$ 发现, 底栖杂食性鱼类鲫降低 了束丝藻的比例, 他们认为鱼类引起的沉积物再悬浮是引起束丝藻比例下降的主要原因, 因为浮游藻类的 比例与水体 ISS 浓度呈显著负相关关系 ${ }^{[42]}$. 而引起研究结果存在差异的主因可能主要与鳑鲏对 ISS 的浓度 无显著影响有关.

\section{3 大鯺鱊对底栖动物的影响分析}

底栖杂食性鱼类, 可降低底栖动物生物量 ${ }^{[3,43-44]}$. 然而, 鳑鲏并未显著影响底栖动物生物量, 反而鳑鲏 组的底栖动物总丰度显著高于对照组. 水丝蚓是鳑鲏组底栖动物群落的优势种. 在前面的讨论中, 通过比 较鳑鲏组与对照组间的 ISS 浓度差异认为鳑鲏对沉积物再悬浮的作用较弱, 这也反映了鳑鲏几乎不在沉积 物表面摄食, 从而对水丝蚓的摄食压力较弱, 促进了水丝蚓的种群发展. 但这与底栖杂食性鱼类鲫对底栖 动物生物量产生的影响不同, $\mathrm{He}$ 等 $^{[3]}$ 发现鲫可显著降低底栖动物生物量. 此外, 鳑鲏组水丝蚓丰度升高可 能还与鳑鲏引起的较高浮游植物生物量有关. 例如 Cai 等 ${ }^{[45-46]}$ 的野外调查发现巢湖和太湖的水丝蚓生物量 随浮游藻类生物量的增加而上升. 在原位受控实验中, 研究人员也发现浮游植物碎屑是水丝蚓的重要食物 来源 ${ }^{[47-48]}$, 大量的浮游藻类碎屑可显著促进水丝蚂生物量增加 ${ }^{[49]}$. 因此, 鳑鲏对水丝蚓较弱的捕食压力和 鳑鲏驱动的较高初级生产力 (浮游植物生物量上升) 是引起底栖动物丰度显著高于对照组的主要原因.

\section{4 河蛙对水质及生物群落的影响分析}

河蚌通过排泄可引起水体营养盐浓度升高 ${ }^{[50-51]}$, 而本研究中河蚌 (河蚌组) 未显著影响水体的氮、磷浓 度, 说明在本实验中河蚌排泄对水体营养盐的影响较弱. 河蚌通过滤食可显著降低水体悬浮质浓度 ${ }^{[18]}$ 和浮 游植物的生物量 ${ }^{[52]}$. 本研究中, 河蛙 (河蚌+鳑鲏组) 抑制了鳑鲏引起的水体营养盐浓度升高和浮游植物生 物量上升的趋势, 降低了蓝藻在浮游植物群落中的占比. 但河蚌组的悬浮质和 Chl. $a$ 浓度与对照组间无显 著差异, 这可能与河蚌在清水态系统中对悬浮质和浮游植物生物量的影响较弱有关 ${ }^{[18]}$. 底栖滤食性双壳类 通过滤食浮游植物降低浮游动物的食物可得性和直接滤食浮游动物等途径, 可显著影响浮游动物的生物 量 ${ }^{[53]}$, 而本研究中背角无齿蚌并未显著影响浮游动物的丰度和生物量; 而降低了枝角类和桡足类的生物 量, 这与斑马贻贝等双壳类的研究结果一致 ${ }^{[54]}$.

\section{5 对浅水湖泊生态修复的启示}

本研究对浅水湖泊生态修复具有一定的指导意义. 鳑鲔等小型杂食性鱼类种群在湖泊生态修复后恢复 较快, 在肉食性鱼类捕食压力较弱时易发展为草型湖泊的优势鱼类 ${ }^{[8]}$. 鳑鲔可引起水体营养盐浓度升高和 浮游动物小型化, 从而促进浮游植物生长, 导致蓝澡发展成为优势种, 且这些负面影响随鳑鲏密度的升高 而增强 ${ }^{[49]}$. 因此, 鳑鲏对水生态系统产生的这些影响将直接或间接地影响沉水植物生长与繁殖. 此外, 鳑 鲏还可直接摄食沉水植物 ${ }^{[8]}$. 在本研究中, 虽然河蚌可削弱鳑鲏的这些负面影响, 但当鳑鲏密度逐渐升高 时, 这种抵消作用可能会逐渐减弱. 因此, 在湖泊生态修复和管理中, 需监测鳑鲏等小型杂食性鱼类密度, 关注小型杂食性鱼类对水生态系统可能产生的负面影响. 为了达到生态修复效果长效与稳定运行的目标, 根据本文的研究结果, 建议通过物理 (网穊等) 和生物 (如放养有效地肉食性鱼类) 等方式将此类小型鱼类 控制在较低的生物量水平.

\section{4 结论}

1) 大鳍魣(鳑鲏)引起 TN、TP、悬浮质和 Chl. $a$ 浓度升高, 而河蚌削弱了鳑鲏的这些负面影响. 鳑鲏与 河蚌交互作用对 TP 和 Chl. $a$ 浓度的影响显著.

2) 鳑鲏 (鳑鲏组) 促进了浮游植物丰度和生物量增加, 其中蓝藻门的束丝藻是浮游植物群落的优势种. 河蚌 (鳑鲏+河蚌组) 降低了浮游植物丰度、生物量和蓝藻的比例. 
3) 鳑鲏显著增加了浮游动物总丰度, 而对浮游动物总生物量的影响不显著; 鳑鲏引起浮游动物群落 以小型种类 (轮虫) 为主, 导致枝角类和桡足类生物量下降. 鳑鲏与河蛙交互作用对浮游动物丰度的影响显 著, 而对生物量无显著影响.

4) 鳑鲏未显著影响底栖动物的生物量, 但促进了底栖动物丰度升高 (以水丝蚓为主).

5) 背角无齿蚌抵消了鳑鲏对水质造成的部分负面影响. 主要表现为: 河蚌降低了水体营养盐和悬浮质 浓度, 引起 Chl. $a$ 和浮游植物生物量下降; 但河蛙对浮游动物和底栖动物生物量的影响不显著.

致谢：感谢沈睿杰对受控实验的支持.

\section{5 参考文献}

[ 1 ] Gu J, Jin H, He H et al. Effects of small-sized crucian carp (Carassius carassius) on the growth of submerged macrophytes: Implications for shallow lake restoration. Ecological Engineering, 2016, 95: 567-573. DOI: 10.1016/j. ecoleng. 2016.06.118.

[ 2 ] Zhang XF, Mei XY, Gulati RD. Effects of omnivorous tilapia on water turbidity and primary production dynamics in shallow lakes: Implications for ecosystem management. Reviews in Fish Biology and Fisheries, 2017, 27(1) : 245-254. DOI: $10.1007 / \mathrm{s} 11160-016-9458-6$.

[ 3 ] He H, Han YQ, Li QS et al. Crucian carp (Carassius carassius) strongly affect C/N/P stoichiometry of suspended particulate matter in shallow warm water eutrophic lakes. Water, 2019, 11(3) : 524. DOI: 10.3390/w11030524.

[ 4 ] Qiu X, Mei XY, Vladimir R et al. Effects of common carp (Cyprinus carpio) on water quality in aquatic ecosystems dominated by submerged plants: a mesocosm study. Knowledge \& Management of Aquatic Ecosystems, 2019, 420: 28. DOI: 10. $1051 / \mathrm{kmae} / 2019017$.

[ 5 ] He H, Liu XB, Liu XL et al. Effects of cyanobacterial blooms on submerged macrophytes alleviated by the native Chinese bivalve Hyriopsis cumingii: A mesocosm experiment study. Ecological Engineering, 2014, 71: 363-367. DOI: 10.1016/j. ecoleng.2014.07.015.

[ 6 ] He H, Hu E, Yu JL et al. Does turbidity induced by Carassius carassius limit phytoplankton growth? A mesocosm study. Environmental Science and Pollution Research, 2017, 24(5) : 5012-5018. DOI: 10.1007/s11356-016-8247-z.

[ 7 ] Zhang XF, Liu ZW, Jeppesen E et al. Effects of benthic-feeding common carp and filter-feeding silver carp on benthic-pelagic coupling: implications for shallow lake management. Ecological Engineering, 2016, 88: 256-264. DOI: 10. 1016/j. ecoleng.2015.12.039.

[ 8 ] Yu JL, Liu ZW, He H et al. Submerged macrophytes facilitate dominance of omnivorous fish in a subtropical shallow lake: implications for lake restoration. Hydrobiologia, 2016, 775: 97-107. DOI: 10.1007/s10750-016-2717-7.

[ 9 ] Zhu YR, Liu HZ. Genetic diversity and the biogeographical process of Acheilognathus macropterus revealed by sequence variations of mitochondrial cytochrome b gene. Frontiers of Biology in China, 2007, 2 : 450-455. DOI: 10.1007/s11515007-0069-4.

[10] Koutrakis ET, Kokkinakis AK, Tsikliras AC et al. Characteristics of the European bitterling Rhodeus amarus (Cyprinidae) in the Rihios river, Greece. Journal of Freshwater Ecology, 2003, 18: 615-624. DOI: 10.1080/02705060.2003.9664003.

[11] Vanni MJ, Bowling AM, Dickman EM et al. Nutrient cycling by fish supports relatively more primary production as lake productivity increases. Ecology, 2006, 87: 1696-1709. DOI: 10.1890/0012-9658(2006) 87[ 1696: NCBFSR]2.0.CO;2.

[12] Gu J, He H, Jin H et al. Synergistic negative effects of small-sized benthivorous fish and nitrogen loading on the growth of submerged macrophytes-relevance for shallow lake restoration. Science of the Total Environment, 2018, 610: 1572-1580. DOI: $10.1016 /$ j.scitotenv.2017.06.119.

[13] Yu Q, Wang J, Liu, Y. Effects of intensive Rhodeus sinensis culture on both nutritive salts and phytoplankton of water body. Acta Agriculturae Shanghai, 2014, 30(4) : 52-56. [喻倩, 王金庆, 刘娅琴等. 高密度养殖鱼鳑鲏鱼对水体营养 盐浓度和浮游植物群落的影响. 上海农业学报, 2014, 30(4): 52-56.]

[14] Yu JL, Xia ML, Kong M et al. A small omnivorous bitterling fish (Acheilognathus macropterus) facilitates dominance of cyanobacteria, rotifers and Limnodrilus in an outdoor mesocosm experiment. Environmental Science and Pollution Research, 2020, 27 : 23862-23870. DOI: 10.1007/s11356-020-08774-5.

[15] Shen JZ. Experimental investigations into some reproduction aspects of Chinese bitterling, Rhodeus sinensis. Journal of 
Huazhong Agricultural University, 2000, 19(5) : 494-496. [沈建忠. 中华鳑鲏 Rhodeus sinensis 繁殖习性的初步观察. 华中农业大学学报, 2000, 19(5): 494-496.]

[16] Reynolds JD, Debuse VJ, Aldridge DC. Host specialisation in an unusual symbiosis: European bitterlings spawning in freshwater mussels. Oikos, 1997, 78(3) : 539. DOI: 10.2307/3545615.

[17] Smith C, Reynolds JD, Sutherland WJ et al. Adaptive host choice and avoidance of superparasitism in the spawning decisions of bitterling (Rhodeus sericeus). Behavioral Ecology and Sociobiology, 2000, 48 ( 1 ): 29-35. DOI: 10. 1007/s002650000212.

[18] Wu ZK, Qiu XC, Zhang XF et al. Effects of Anodonta woodiana on water quality improvement in restoration of eutrophic shallow lakes. J Lake Sci, 2018, 30(6) : 1610-1615. DOI: 10.18307/2018.0612. [吴中奎, 邱小常, 张修峰等. 富营 养化浅水湖泊生态修复中背角无齿蚌 (Anodonta woodiana) 对水质改善的影响. 湖泊科学, 2018, 30(6): 1610-1615.]

[19] Johengen TH, Nalepa TF, Fahnenstiel GL et al. Nutrient changes in Saginaw bay, lake Huron, after the establishment of the zebra mussel (Dreissena polymorpha). Journal of Great Lakes Research, 1995, 21 (4) : 449-464. DOI: 10.1016/ S0380-1330 ( 95 ) 71058-9.

[20] Yang DM, Chen YW, Liu ZW et al. Top-down effects of Anodonta woodiana on nutrient concentration \& phytoplankton community composition in a microcosm ecosystem. J Lake Sci, 2008, 20(2) : 228-234. DOI: 10.18307/2008.0215. [杨 东妹, 陈宇炜, 刘正文等. 背角无齿蚌滤食对营养盐和浮游藻类结构影响的模拟. 湖泊科学, 2008, 20 (2): 228-234. ]

[21] Gao HL, Qian X, Wu HF et al. Combined effects of submerged macrophytes and aquatic animals on the restoration of a eutrophic water body-A case study of Gonghu Bay, Lake Taihu. Ecological Engineering, 2017, 102 : 15-23. DOI: 10. 1016/j. ecoleng.2017.01.013.

[22] Qin BQ. A large-scale biological control experiment to improve water quality in eutrophic Lake Taihu, China. Lake and Reservoir Management, 2013, 29(1) : 33-46. DOI: 10.1080/10402381.2013.767867.

[23] Liu ZW, Hu JR, Zhong P et al. Successful restoration of a tropical shallow eutrophic lake: Strong bottom-up but weak topdown effects recorded. Water Research, 2018, 146: 88-97. DOI: 10.1016/j.watres.2018.09.007.

[24] Atkinson CL, Vaughn CC. Biogeochemical hotspots: Temporal and spatial scaling of the impact of freshwater mussels on ecosystem function. Freshwater Biology, 2015, 60(3) : 563-574. DOI: 10.1111/fwb.12498.

[25] Mills SC, Reynolds JD. The bitterling-mussel interaction as a test case for co-evolution. Journal of Fish Biology, 2003, 63: 84-104. DOI: 10.1111/j.1095-8649.2003.00209.x.

[26] Reichard M, Polačik M, Tarkan AS et al. The bitterling-mussel coevolutionary relationship in areas of recent and ancient sympatry. Evolution, 2010, 64(10) : 3047-3056. DOI: 10.1111/j.1558-5646.2010.01032.x.

[27] Ye SW, Li ZJ, Lek-Ang S et al. Community structure of small fishes in a shallow macrophytic lake (Niushan Lake) along the middle reach of the Yangtze River, China. Aquatic Living Resources, 2006, 19(4): 349-359. DOI: 10. 1051/ alr: 2007005.

[28] Cai YJ, Gong ZJ, Qin BQ. Standing crop and spatial distributional pattern of Mollusca in Lake Taihu, 2006-2007. J Lake Sci, 2009, 21 (5) : 713-719. DOI: 10.18307/2009. 0516. [蔡永久, 龚志军, 秦伯强. 太湖软体动物现存量及空间分 布格局(2006-2007 年). 湖泊科学, 2009, 21(5) : 713-719.]

[29] Hu HJ, Wei YX eds. The freshwater algae of China: Systematics, taxonomy and ecology. Beijing: Science Press, 2006. [胡鸿钧, 魏印心. 中国淡水藻类——系统、分类及生态. 北京: 科学出版社, 2006.]

[30] Guiry MD, Guiry GM eds. AlgaeBase. Galway: World-wide electronic publication, National University of Ireland, 2017. URL: http://www. algaebase.org.

[31] Reynolds RS ed. The ecology of freshwater phytoplankton. Cambridge: Cambridge University Press, 1984.

[32] Jiang XZ, Du NS eds. Fauna sinica, crustacea, freshwater cladocera. Beijing: Science Press, 1979. [蒋繂治, 堵南山. 中国动物志. 节肢动物门，甲壳纲，淡水枝角类. 北京: 科学出版社, 1979.]

[33] Shen JR ed. Fauna sinica, crustacea, freshwater copepoda. Beijing: Science Press, 1979. [沈嘉瑞. 中国动物志. 节肢 动物门, 甲壳纲, 淡水桡足类. 北京: 科学出版社, 1979.]

[34] Dumont HJ, Velde I, Dumont S. The dry weight estimate of biomass in a selection of Cladocera, Copepoda and Rotifera from the plankton, periphyton and benthos of continental waters. Oecologia, 1975, 19 ( 1): 75-97. DOI: 10. 
1007/BF00377592.

[35] Huang XF ed. Survey observation and analysis of lake ecology. Beijing: China Standard Press, 1999. [黄祥飞. 湖泊生态 调查观测与分析. 北京: 中国标准出版社, 1999.]

[36] Jin XC, Tu QY eds. The standard methods in lake eutrophication investigation. Beijing: China Environmental Science Press, 1990. [ 金相灿, 屠清瑛. 湖泊富营养化调查规范. 北京: 中国环境科学出版社, 1990.]

[37] Attayde JL, Hansson LA. The relative importance of fish predation and excretion effects on planktonic communities. Limnology and Oceanography, 2001, 46(5) : 1001-1012. DOI: 10.4319/lo.2001.46.5.1001.

[38] Brabrand $\AA$, Faafeng BA, Moritz NJP. Relative importance of phosphorus supply to phytoplankton production: Fish excretion versus external loading. Canadian Journal of Fisheries and Aquatic Sciences, 1990, 47(2) : 364-372. DOI: 10.1139/ f90-038.

[39] Brooks JL, Dodson SI. Predation, body size, and composition of plankton. Science, 1965, 150(3692) : 28-35. DOI: 10. 1126/science.150.3692.28.

[40] Havens KE. Fish-induced sediment resuspension: Effects on phytoplankton biomass and community structure in a shallow hypereutrophic lake. Journal of Plankton Research, 1991, 13(6) : 1163-1176. DOI: 10.1093/plankt/13.6.1163.

[41] Jeppesen E, Søndergaard M, Lauridsen TL et al. Biomanipulation as a restoration tool to combat eutrophication. Advances in ecological research. Amsterdam: Elsevier, 2012 : 411-488. DOI: 10.1016/b978-0-12-398315-2.00006-5.

[42] Roozen FCJM, Lürling M, Vlek H et al. Resuspension of algal cells by benthivorous fish boosts phytoplankton biomass and alters community structure in shallow lakes. Freshwater Biology, 2007, 52 (6) : 977-987. DOI: 10.1111/j.1365-2427. 2007.01729.x.

[43] Miller SA, Crowl TA. Effects of common carp (Cyprinus carpio) on macrophytes and invertebrate communities in a shallow lake. Freshwater Biology, 2006, 51(1) : 85-94. DOI: 10.1111/j.1365-2427.2005.01477.x.

[44] Weber MJ, Brown ML. Effects of common carp on aquatic ecosystems 80 years after “carp as a dominant”: Ecological insights for fisheries management. Reviews in Fisheries Science, 2009, 17 ( 4 ): 524-537. DOI: 10. 1080/10641260903189243.

[45] Cai YJ, Gong ZJ, Qin BQ. Benthic macroinvertebrate community structure in Lake Taihu, China: Effects of trophic status, wind-induced disturbance and habitat complexity. Journal of Great Lakes Research, 2012, 38(1) : 39-48. DOI: 10.1016/ j.jglr.2011.12.009.

[46] Cai Y, Gong Z, Xie P. Community structure and spatiotemporal patterns of macrozoobenthos in Lake Chaohu (China). Aquatic Biology, 2012, 17(1) : 35-46. DOI: 10.3354/ab00455.

[47] Yu JL, Li YM, Liu XL et al. The fate of cyanobacterial detritus in the food web of Lake Taihu: A mesocosm study using ${ }^{13} \mathrm{C}$ and ${ }^{15} \mathrm{~N}$ labeling. Hydrobiologia, 2013, 710(1) : 39-46. DOI: 10.1007/s10750-012-1205-y.

[48] Yu JL, He H, Liu ZW et al. Carbon transfer from cyanobacteria to pelagic and benthic consumers in a subtropical lake: Evidence from a ${ }^{13} \mathrm{C}$ labelling experiment. Water, 2019, 11(8) : 1536. DOI: 10.3390/w11081536.

[49] Xia ML, Yu JL, He Het al. Effects of cyanobacterial detritus on water quality and the biomass of periphyton and Limnodrilus spp. in Lake Taihu. J Lake Sci, 2020, 32(4) : 1050-1059. DOI: 10.18307/2020.0414. [夏曼莉, 于谨否, 何虎等. 太湖蓝藻碎屑对水质及附着藻和水丝蚂生物量的影响. 湖泊科学, 2020, 32(4): 1050-1059.]

[50] Coelho JP, Lillebø AI, Crespo D et al. Effect of the alien invasive bivalve Corbicula fluminea on the nutrient dynamics under climate change scenarios. Estuarine, Coastal and Shelf Science, 2018, 204: 273-282. DOI: 10.1016/j. ecss.2018. 03.001 .

[51] Parr TB, Vaughn CC, Gido KB. Animal effects on dissolved organic carbon bioavailability in an algal controlled ecosystem. Freshwater Biology, 2020, 65(7) : 1298-1310. DOI: 10.1111/fwb.13438.

[52] Zhang XF, Liu ZW, Jeppesen E et al. Effects of deposit-feeding tubificid worms and filter-feeding bivalves on benthic-pelagic coupling: Implications for the restoration of eutrophic shallow lakes. Water Research, 2014, 50: 135-146. DOI: 10. 1016/j.watres.2013.12.003.

[53] Jack JD, Thorp JH. Effects of the benthic suspension feeder Dreissena polymorpha on zooplankton in a large river. Freshwater Biology, 2000, 44(4) : 569-579. DOI: 10.1046/j.1365-2427.2000.00609.x.

[54] Thorp JH, Casper AF. Potential effects on zooplankton from species shifts in planktivorous mussels: A field experiment in the St Lawrence River. Freshwater Biology, 2002, 47(1) : 107-119. DOI: 10.1046/j.1365-2427.2002.00787.x. 